\title{
Dynamic quasi concave performance measures
}

\author{
Sara Biagini * Jocelyne Bion-Nadal ${ }^{\dagger}$
}

October 25, 2018

\begin{abstract}
We define Conditional quasi concave Performance Measures (CPMs), on random variables bounded from below, to accommodate for additional information. Our notion encompasses a wide variety of cases, from conditional expected utility and certainty equivalent to conditional acceptability indexes. We provide the characterization of a CPM in terms of an induced family of conditional convex risk measures. In the case of indexes these risk measures are coherent. Then, Dynamic Performance Measures (DPMs) are introduced and the problem of time consistency is addressed. The definition of time consistency chosen here ensures that the positions which are considered good tomorrow are already considered good today. We prove the equivalence between time consistency for a DPM and weak acceptance consistency for the induced families of risk measures. Finally, we extend CPMs and DPMs to dividend processes.
\end{abstract}

Key words: Conditional performance measure, conditional acceptability index, induced family of risk measures, dynamic performance measure, time consistency, risk to reward ratio

JEL Classification: primary D81, secondary G11

MSC 2010 Classification: primary 91B16 and 91B30, secondary 91B70, 46B42

\section{Introduction}

Portfolio selection and the companion asset allocation are undoubtedly one of the most important problems in Finance and Insurance. In order to make an optimal choice, some performance criterion must be selected. Since Markowitz's seminal work many criteria have been proposed, from expected utility to ratios which seek a balance between reward and risk, such as the Sharpe Ratio index. The Sharpe Ratio of a position consists of

\footnotetext{
*University of Pisa, Italy

${ }^{\dagger}$ CNRS, CMAP - Ecole Polytechnique, France
} 
the ratio of expected value over standard deviation. It has many pleasant features: clear meaning, easiness of computation, scale invariance and has consequently had a great success in the industry. From a normative point of view, and outside a Gaussian context, it is well-known that this index has many drawbacks: it is not monotone and it may seriously underestimate arbitrage (see e.g. Bernardo and Ledoit [3, Section 2.2.1]). Thus, a whole class of indexes have been developed to improve the Sharpe Ratio in investment evaluation, while preserving its good features. Some examples are the Sortino Ratio, the Gain-Loss Ratio by Bernardo and Ledoit, or, more generally, the ratio of reward to lower p-th partial moments in Leitner [21], and generalized Sharpe Ratios by Černý [12].

More recently, Cherny and Madan [15] have built a theoretical framework for these $a c$ ceptability indexes. Their axiomatic definition stems from an analysis of the theoretically and practically desirable properties a performance criterion should possess. For bounded positions, they show a complete characterization of an acceptability index in terms of a parametric family of coherent risk measures and propose some new law invariant indexes. The setup in [15] is static, in the sense that there are only two dates of interest: today and the horizon $T$, and consequently the index is real valued - possibly infinite.

Dynamic acceptability indexes and their dual representations have been thoroughly analyzed in Bielecki at alii [6], in a finite $\Omega$, finite set of dates $\mathcal{T}$ framework. Rosazza Gianin and Sgarra 24] remove the requirement of scale invariance and provide a (static) characterization of these generalized acceptability indexes in terms of a family of quasi convex risk measures on $L^{\infty}$. Then, they work in a Brownian context and focus on dynamic acceptability indexes generated by $g$-expectations and give applications to liquidity risk quantification.

Our purpose is to deepen the theoretical analysis of dynamic performance criteria. In Section 2, over a general probability space and stochastic basis, we define a Conditional Performance Measure (CPM) for positions bounded from below.

The fact that the domain of the CPM includes not only bounded positions but also those bounded from below leads to reconsider the continuity axioms. While the current literature requires an index to be continuous from above, we ask for continuity from below.

Thus, we come out with a performance criterion which encompasses conditional expected utility and conditional acceptability indexes as a particular cases, as shown in the Examples in Section 2.1. Then, in Section 3 we provide the representation of a CPM in terms of an induced family of risk measures. Such family is in fact convex, and not only quasi convex, so we complete and extend to the conditional case the intuition for the static case in [24, Proposition 3]. The results in Section 3 are, in order:

- in Section 3.1, we prove a CPM $\beta_{t}$ induces a parametric family of convex risk measures $\left(\rho_{t}^{z}\right)_{z}$, with $z \in\left(z_{d}, z_{u}\right)$ where $z_{d}<z_{u}$ are the (non-random) essential infimum and supremum of the CPM. The properties of $\left(\rho_{t}^{z}\right)_{z}$ are listed in Proposition 3.3 , 
- in Section 3.2 we define standard families of risk measures, and we show that any such family generates a CPM;

- $\left(\rho_{t}^{z}\right)_{z}$ is the unique standard family which generates $\beta_{t}$. This is shown in Proposition 3.12 .

In Section 4, the dynamic case is considered. A Dynamic Performance Measure (DPM) is naturally defined as a collection of CPMs and the time consistency problem is addressed. The notion we choose is very close to the one in [24, Proposition 6], and basically ensures that positions which are considered good tomorrow are already considered good today. In virtue of the results in Section 3, a time consistency requirement on the DPM must have an equivalent in terms of the time consistency of the induced families of convex risk measures. In fact, Proposition 4.4 shows our notion of time consistency for a DPM is equivalent to the weak acceptance consistency for the induced risks. We conclude with some examples and counterexamples on DPMs (Subsection 4.2), including DPM generated by $g$-expectations, and with a note on the extension of CPMs and DPMs to dividend processes (Subsection 4.3).

\section{Conditional performance measures and accept- ability indexes}

A fixed time horizon $T>0$ is given and the trading dates $\mathcal{T}$ are a subset of $[0, T]$, typically either a finite subset $\left\{t_{1}, t_{2}, \ldots t_{n}\right\}, t_{n}=T$, or the entire interval. The stochastic basis $\left(\Omega,\left(\mathcal{F}_{t}\right)_{t \in \mathcal{T}}, P\right)$ models the possible outcomes, the evolution of the information with time and the probability of the various events. In the following, $t$ is always assumed to be in $\mathcal{T}$. For a shorthand, let us denote by $L_{t}^{\infty}$ the space $L^{\infty}\left(\Omega, \mathcal{F}_{t}, P\right)$ of essentially bounded variables and let $L_{t}^{b b}$ denote the lattice of essentially bounded from below ones, possibly $+\infty$-valued. Equalities and inequalities are intended to hold $P$-almost surely. For example, $X>0$ means $P(X>0)=1$, and we refer to elements of $L_{t}^{b b}$ simply as 'bounded from below' variables and to elements in $\mathrm{E}_{t}^{\infty}$ as 'bounded'. The space of simple variables in $L_{t}^{b b}$, i.e. those $\mathbb{R}$-valued and assuming only a finite set of real values, is denoted by $\mathcal{S}_{t}$. The conditional expectations $E\left[\cdot \mid \mathcal{F}_{t}\right], E^{Q}\left[\cdot \mid \mathcal{F}_{t}\right]$ where $Q \ll P$ are denoted by $E_{t}[\cdot], E_{t}^{Q}[\cdot]$.

The convention adopted hereafter is that notation is self-explaining. (Equivalence classes of $\mathcal{F}_{T}$-measurable random variables are denoted by capital Latin letters $X, Y, \ldots$, $\mathcal{F}_{t}$-measurable random variables by the Greek letters $\xi, \eta$, while the Greeks $\varphi, \psi$ are re-

served for elements of $\mathcal{S}_{t}$. Real numbers will be indicated by lower Latin letters $a, b, c, d \ldots$ However, for clarity's sake there will be complete statements like e.g. $c \in \mathbb{R}$ in the definitions and where any misunderstanding is possible. 
Definition 2.1. A performance measure, conditional to $\mathcal{F}_{t}$, is a map $\beta_{t}: L_{T}^{b b} \rightarrow L_{t}^{b b}$ with the following six properties:

1. quasi-concavity: for any real $c \in[0,1]$ and for any $X, Y \in L_{T}^{b b}$

$$
\beta_{t}(c X+(1-c) Y) \geq \min \left(\beta_{t}(X), \beta_{t}(Y)\right)
$$

2. if $z_{d}, z_{u}$ denote the infimum and supremum of $\beta_{t}$,

$$
z_{d}=\operatorname{ess} \inf _{X} \beta_{t}(X), z_{u}=\operatorname{ess} \sup _{X} \beta_{t}(X)
$$

then, $z_{d}, z_{u}$ are non-random and $z_{d}<z_{u}$ (possibly infinite);

3. monotonicity: $\beta_{t}$ is

(a) non decreasing, $\beta_{t}(X) \geq \beta_{t}(Y)$ whenever $X \geq Y$;

(b) increasing over constant positive shifts:

$$
c>0 \Rightarrow \beta_{t}(X+c)>\beta_{t}(X) \text { on }\left\{\beta_{t}(X)<z_{u}\right\} \cap\left\{\beta_{t}(X+c)>z_{d}\right\}
$$

4. continuity from below: if $X_{n} \uparrow X \in L_{T}^{b b}$, then $\beta_{t}(X)=\lim _{n} \beta_{t}\left(X_{n}\right)$;

5. locality:

$$
I_{B} \beta_{t}(X)=I_{B} \beta_{t}\left(X I_{B}\right) \forall B \in \mathcal{F}_{t}
$$

6. for each $z \in \mathbb{R}$, let $A_{t}^{z}:=\left\{X \in L_{T}^{b b} \mid \beta_{t}(X) \geq z\right\}$ denote the z-upper level set of $\beta_{t}$. If $z_{d}<z<z_{u}$, then $A_{t}^{z} \cap L_{t}^{b b}$ is uniformly bounded from below, i.e. ess inf $A_{t}^{z} \cap L_{t}^{b b} \geq x_{z}$ for some $x_{z} \in \mathbb{R}$.

For a shorthand, conditional performance measures will be indicated by the acronym CPM. If the CPM further satisfies:

7. scale invariance: the map is positively homogeneous of degree 0 ,

$$
\beta_{t}(c X)=\beta_{t}(X), \text { for all } X \text { and reals } c>0
$$

8. nonnegativity: $\beta_{t} \geq 0$,

it is called Conditional Acceptability Index (CAI).

Some of the above properties are intuitive: quasi-concavity means diversification is encouraged by the CPM, monotonicity that more is preferred to less. The requirement of non-randomness on $z_{d}, z_{u}$ and that of strict monotonicity on constant positive shifts are technical, but quite reasonable and verified in all the examples we will see. 
Continuity from below, Property 4, in turn ensures that profiles which are 'pointwiseclose' to a given profile $X$ in the limit cannot underperform $X$.

To give a precise mathematical statement for the last assertion, recall first the notion of order convergence in a vector lattice $L$. A sequence $\left(X_{n}\right)_{n}$ is order convergent to $X$, notation $X_{n} \stackrel{o}{\rightarrow} X$ if there exists a nonnegative sequence $\left(Y_{n}\right)_{n}$ such that

$$
\left|X_{n}-X\right| \leq Y_{n} \text { and } Y_{n} \downarrow 0
$$

We refer to Aliprantis and Border [2, Chapters 8-9], for more details on lattices and order convergence, to Foellmer and Schied [17, Section 4.2] for applications of these notions to convex risk measures on spaces of bounded variables, and to Biagini and Frittelli [5] when the convex risk measures are defined over general spaces.

The following Lemma is the analog of the equivalence between the Monotone Convergence Theorem and Fatou Lemma in Calculus. An immediate corollary is that for a $\mathrm{CPM} \beta_{t}: L_{T}^{b b} \rightarrow L_{t}^{b b}$ continuity from below is equivalent to order lower semicontinuity with respect to sequences uniformly bounded from below.

Lemma 2.2. With the convention $\infty-\infty=0$ and $c+\infty=\infty$, for a monotone non decreasing map $\pi: L_{T}^{b b} \rightarrow L_{t}^{b b}$ continuity from below is equivalent to order lower semicontinuity with respect to sequences uniformly bounded from below, i.e. is equivalent to:

$$
X_{n} \stackrel{o}{\rightarrow} X \text { and } X_{n} \geq c \text { for all } n \Rightarrow \pi(X) \leq \lim _{n} \inf \pi\left(X_{n}\right)
$$

Proof. $L_{T}^{b b}, L_{t}^{b b}$ are only lattices and not vector spaces, but any absolute difference $|Y-X|$ is a well defined element of $L_{T}^{b b}$ under the stated convention. The only thing to prove is that continuity from below implies order lsc with respect to sequences bounded from below. Assume then $\pi$ is continuous from below and pick a sequence $\left(X_{n}\right)_{n}, X_{n} \geq c$, which is order convergent to $X$; then, $X_{n} \rightarrow X$ a.s., so $X \geq c$, and there exists a sequence $Y_{n} \downarrow 0$ such that $\left|X_{n}-X\right| \leq Y_{n}$. So,

$$
\left(X-Y_{n}\right) \vee c \leq X_{n} \text { and }\left(X-Y_{n}\right) \vee c \uparrow X
$$

whence, passing to the liminf, by monotonicity and continuity from below:

$$
\pi(X)=\lim _{n} \pi\left(\left(X-Y_{n}\right) \vee c\right) \leq \liminf _{n} \pi\left(X_{n}\right)
$$

Note that the monotonicity of $\beta_{t},+\infty \in L_{T}^{b b}$ and continuity from below of $\beta_{t}$ imply

$$
z_{u}=\beta_{t}(+\infty)=\lim _{x \uparrow+\infty} \beta_{t}(x)
$$

Since $\beta_{t}$ is defined on $L_{T}^{b b}$, monotonicity also implies

$$
z_{d}=\lim _{x \downarrow-\infty} \beta_{t}(x)
$$


Therefore, for any $z_{d} \leq z<z_{u}$, the upper level set $A_{t}^{z}$ contains a constant. This will be often used in the following, in the form

$$
z_{d} \leq z<z_{u} \Rightarrow A_{t}^{z} \cap L_{t}^{\infty} \neq \emptyset
$$

Property 5 , locality, is rather natural. On a set of states $B$ revealed at $t$, the performance of the future profile $X$ is uniquely determined by the restriction of $X$ to $B$, all the other states being irrelevant.

The last property of CPM, property 6, is a mild technical assumption, as it is verified in a number of interesting cases as shown in the Examples in Section 2.1. Finally, scale invariance and nonnegativity are requirements for (static) acceptability indexes as introduced by Cherny and Madan [15]. Scale invariance for a performance means it evaluates the goodness of the whole direction of trade generated by a profile $X$, namely its ray, rather than $X$ only. This may be appropriate only for a large investor, for whom quantity does not matter, and when the liquidity risk is negligible.

Remark 2.3. The motivations supporting the choice of continuity from below for $\beta_{t}$, instead of continuity from above as assumed in [15, 24, 6], are manifold.

1. Some natural performance measures as conditional expectation/expected utility/certainty equivalent on $L_{T}^{b b}$ are not continuous from above, but are continuous from below.

2. Continuity from below solves the value-at-0 puzzle for indexes. In the set up of Cherny and Madan, continuity from above implies that an index unbounded above must be necessarily $+\infty$ in 0 . This is rather awkward for any index, but especially for those null on the negative constants or on the whole negative orthant. In our context an index null on negative constants in 0 is naturally 0 valued. See Lemma 2.4 and Remark 2.5 for a proof of this simple fact, and Section 2.1 for the concrete example of the Bernardo and Ledoit's Gain-Loss Ratio index and the Best Gain-Loss Ratio index introduced by Biagini and Pinar [4].

3. As shown in Section 3, continuity from below of $\beta_{t}$ implies the associated risk measures $\rho_{t}^{z}$ are also continuous from below. This in turn implies that the risks, when restricted to $L^{\infty}$, have a special dual representation which implies continuity from above on $L^{\infty}$. This special dual representation, with a maximum over the dual variables, is essential in any practical application. See e.g. the examples given by [15].

4. Last, but not least, in many specific cases there is a natural vector space (a Banach lattice in fact) over which the map can be defined. This space is $L^{1}$ for the case of Gain-Loss Ratio, $L^{p}, p<+\infty$ for Lower Partial Moments analysis, or, more generally, an Orlicz heart as in the Examples 2.9, 2.10 and 2.11. If the map is 
e.g. a (quasi concave) ratio of concave monotone increasing function over a convex monotone decreasing function, it will be norm continuous in the interior of its proper domain by the extended Namioka-Klee Theorem. Since these norm topologies are order continuous, the map will be globally order continuous, from above and below, on the interior of the proper domain. See [5] for more details on this important point.

Lemma 2.4. Suppose a $C P M \beta_{t}$ is scale invariant. Then, for all $\xi \in L_{t}^{b b}$

$$
\beta_{t}(\xi)=\beta_{t}(1) I_{\{\xi>0\}}+\beta_{t}(0) I_{\{\xi \leq 0\}}
$$

and consequently $\beta_{t}(X)=\beta_{t}(0)=z_{d}$ for all $X \in L_{T}^{b b}, X \leq 0$ and $\beta_{t}(X)=\beta_{t}(1)=z_{u}$ for all $X$ such that $X \geq \xi>0$ for some $\xi \in L_{t}^{b b}$.

In particular, when $\beta_{t}$ is unbounded above $\left(z_{u}=+\infty\right)$, then $\beta_{t}(X)=+\infty$ on all $X \in L_{T}^{b b}$ such that $X \geq \xi>0$ for some $\xi \in L_{t}^{b b}$.

Proof. Suppose first $\xi>0$ and pick $\varphi_{n} \in \mathcal{S}_{t}, \varphi_{n} \uparrow \xi$. Note $\varphi_{n}$ cannot be chosen strictly positive in general. Since $\varphi_{n}$ is simple, the locality property and scale invariance imply $\beta_{t}\left(\varphi_{n}\right) I_{\left\{\varphi_{n}>0\right\}}=\beta_{t}\left(\varphi_{n} I_{\left\{\varphi_{n}>0\right\}}\right) I_{\left\{\varphi_{n}>0\right\}}=\beta_{t}\left(I_{\left\{\varphi_{n}>0\right\}}\right) I_{\left\{\varphi_{n}>0\right\}}$. By continuity from below then

$$
\beta_{t}(\xi)=\lim _{n} \beta_{t}\left(\varphi_{n}\right)=\lim _{n} \beta_{t}\left(\varphi_{n}\right) I_{\left\{\varphi_{n}>0\right\}}=\lim _{n} \beta_{t}\left(I_{\left\{\varphi_{n}>0\right\}}\right) I_{\left\{\varphi_{n}>0\right\}}=\beta_{t}(1)
$$

On the other side, locality, scale invariance and continuity from below give $\beta_{t}(\varphi)=\beta_{t}(0)$ for all $\varphi \leq 0$ in $S_{t}$. Let $\xi \in L_{t}^{b b}, \xi \leq 0$. Then, $\xi$ is bounded and if $c$ indicates a negative lower bound, by monotonicity $\beta_{t}(c) \leq \beta_{t}(\xi) \leq \beta_{t}(0)$, whence $\beta_{t}(\xi)=0$ and (5) follows by localization. The conclusions for $X \in L_{T}^{b b}$ are easy consequences of (5) and of the monotonicity property.

The last assertion follows from (3) and (5)

Remark 2.5 (On CAIs). Thanks to the above Lemma, a CAI which is: 1) unbounded above; and 2) expectation consistent, i.e. $\beta_{t}(X)=0$ for all $X$ such that $E_{t}[X] \leq 0$ and $\beta_{t}(X)>0$ if $E_{t}[X]>0$, verifies

$$
\beta_{t}(\xi)=+\infty I_{\{\xi>0\}}+0 I_{\{\xi \leq 0\}} \text { on } L_{t}^{b b}
$$

and $\beta_{t}(X)=\beta_{t}(0)=0$ for all $X \in L_{T}^{b b}, X \leq 0$ and $\beta_{t}(X)=+\infty$ for all $X$ such that $X \geq \xi>0$ for some $\xi \in L_{t}^{b b}$.

Locality of the CPM ensures that upper level sets are also local, in the sense explained below.

Lemma 2.6. Let $\beta_{t}$ be a CPM and suppose $X_{1}, X_{2}$ satisfy:

$$
\beta_{t}\left(X_{1}\right) \geq z \text { on } B \in \mathcal{F}_{t}, \beta_{t}\left(X_{2}\right) \geq z \text { on } B^{c} \text {. }
$$

Then $Y=X_{1} I_{B}+X_{2} I_{B^{c}} \in A_{t}^{z}$. 
Proof. From locality of $\beta_{t}$,

$$
I_{B} \beta_{t}(Y)=I_{B} \beta_{t}\left(Y I_{B}\right)=I_{B} \beta_{t}\left(X_{1} I_{B}\right)=I_{B} \beta_{t}\left(X_{1}\right) \geq z I_{B}
$$

Similarly on $B^{c}$ we get $I_{B^{c}} \beta_{t}(Y) \geq z I_{B^{c}}$ and therefore $Y \in A_{t}^{z}$.

Corollary 2.7. If $z_{d} \leq z<z_{u}$ and $\beta_{t}(X) \geq z$ on $B \in \mathcal{F}_{t}$, then there exists $\widetilde{X} \in L_{T}^{b b}$, $\widetilde{X} I_{B}=X I_{B}$ such that $\beta_{t}(\widetilde{X}) \geq z$. The same result holds when inequalities are strict, i.e. if $\beta_{t}(X)>z$ on $B$, then there is $\widetilde{X}$ such that $\beta_{t}(\widetilde{X})>z$.

Proof. When $z<z_{u}, A_{t}^{z} \cap L_{t}^{\infty} \neq \emptyset$ by (4). Pick $\xi$ is this set, let $X_{1}=X, X_{2}=\xi$ and define $\widetilde{X}=Y$ where $Y$ is as in the previous Lemma. For the case of strict inequalities, pick $\xi$ in $A_{t}^{z^{\prime}} \cap L_{t}^{\infty}$ for some $z_{u}>z^{\prime}>z$ and define $\tilde{X}$ in the same way.

\subsection{Examples of CPMs and CAIs}

Example 2.8 (Conditional expected (random) utility). Consider a function $U:(-\infty,+\infty] \times$ $\Omega \rightarrow(-\infty,+\infty]$ which is $\mathcal{B}((-\infty,+\infty]) \otimes \mathcal{F}_{T}$-measurable. We assume it is a stochastic utility function, in the sense that for every fixed $\omega, U(\cdot, \omega)$ is a finite-valued, concave, monotone nondecreasing function. Note $U(\cdot, \omega)$ is a fortiori continuous on $\mathbb{R}$.

In order to define a map via conditional expectation extra assumptions must be imposed. In fact $U(X(\cdot), \cdot)$ is $\mathcal{F}_{T}$ measurable when $X$ is, but conditional expectation may not be either well-defined or $L_{t}^{b b}$-valued on $L_{T}^{b b}$. Also, Properties 2 and 6 of CPMs do not hold for a generic stochastic utility as it can be seen from variations of the items in the examples list below (e.g. if in item 3 the endowment $W$ is not bounded). Suppose then:

a) for all $x \in \mathbb{R}, U(x, \omega) \in L_{T}^{b b}$

b) $U(+\infty, \omega)=\sup _{x} U(x, \omega)$ and $E_{t}[U(+\infty, \omega)] \in(-\infty,+\infty]$

c) for any fixed level $z<z_{u},\left\{\xi \in L_{t}^{b b} \mid E_{t}[U(\xi, \omega)] \geq z\right\}$ contains a bounded variable and is bounded from below.

Define on $L_{T}^{b b}$ :

$$
\beta_{t}(X):=E_{t}[U(X(\omega), \omega)]
$$

Then $\beta_{t}$ is a CPM. In fact, it is easy to verify that item a) implies $\beta_{t}$ is well defined and $L_{t}^{b b}$-valued. This map is evidently concave and monotone. By the monotone convergence Theorem, assumptions a) and b), $\beta_{t}$ is continuous from below (Property 4 of CPM). Assumption b) and monotonicity give:

$$
E_{t}[U(+\infty, \omega)] \geq \operatorname{ess}^{\sup _{X}} E_{t}[U(X(\omega), \omega)] \geq \operatorname{ess}_{\sup _{x}} E_{t}[U(x, \omega)]=E_{t}[U(+\infty, \omega)]
$$


which implies $z_{u}=E_{t}[U(+\infty, \omega)] \in(-\infty,+\infty]$, while assumption c) implies $z_{d}=-\infty$ so that Property 2 of CPMs holds, Property 5 is automatically satisfied given the structure, as

$$
U(X, \omega) I_{B}=U\left(X I_{B}, \omega\right) I_{B}
$$

with the convention $0 \cdot \infty=0$. Property 6 directly follows from assumption $\mathrm{c}$ ), so $\beta_{t}$ is a CPM.

This set up covers, among others, the next cases.

1. Conditional expectation with respect to $Q \ll P, Q_{\mid \mathcal{F}_{t}} \sim P_{\mid \mathcal{F}_{t}}$. Let $Z_{T}:=\frac{d Q}{d P}$, call $Z_{t}=E_{t}\left[Z_{T}\right]$, pick two versions of these variables and set $U(x, \omega)=x \frac{Z_{T}}{Z_{t}}(\omega)$. Assumptions a), b) and c) hold as $E_{t}[U(\cdot, \omega)]=E_{t}^{Q}[\cdot]$ and is thus the identity on $L_{t}^{b b}$.

2. Conditional expected utility. When $U(x, \omega)=U(x)$ and $U(\infty)=\sup _{x} U(x)$, and $\inf _{x} U(x)=-\infty$ we are in the classic, non random utility case. Assumption a) and b) are trivially satisfied. By concavity $U$ is strictly increasing up to $\bar{x}=\inf \{x \mid$ $\left.U(x)=z_{u}=U(+\infty)\right\}$. Then, for any $z<z_{u} U^{-1}$ is well-defined and $E_{t}[U(\xi)]=$ $U(\xi) \geq z$ iff $\xi \geq U^{-1}(z)$, so that $A_{t}^{z} \cap L_{t}^{b b}$ is bounded from below.

3. Conditional expected utility with random endowment $W \in L_{t}^{\infty}$. Here $U(x, \omega)=$ $\widetilde{U}(x+W(\omega))$ where $\widetilde{U}$ is a deterministic utility function as in the previous example. Again assumption a) and b) are easily verified for $\beta_{t}(X)=E_{t}[\widetilde{U}(X+W)]$. Assumption c) is verified as in the previous example.

4. Conditional expected exponential utility with random risk aversion. Let

$$
U(x, \omega)=1-e^{-\gamma(\omega) x}
$$

with $\gamma \in\left(L_{t}^{b b}\right)_{+}, \gamma \geq c>0$. The restrictive measurability assumption on $\gamma$ is due to the financial interpretation as risk aversion is known today, while the uniform lower bound on risk aversion guarantees assumption c) is satisfied.

Example 2.9 (Conditional Certainty Equivalent, CCE). Suppose we are given a stochastic dynamic utility:

$$
U=U(x, t, \omega), t \in \mathcal{T}
$$

where for fixed $t$ the utility satisfies the same conditions as in the previous example and the two extra conditions: $U(\cdot, t, \cdot)=U_{t}$ is $\mathcal{B}(\mathbb{R}) \times \mathcal{F}_{t}$-measurable and $x \rightarrow U(x, t, \omega)$ is a.s. strictly increasing. Fix then $t<T$ and suppose the assumptions a) and b) on $E_{t}[U(\cdot, T, \omega)]$ hold as in the previous example. Then, the CCE at time $t$ is the unique variable $C_{t}(X)$ such that

$$
U\left(C_{t}(X), t, \omega\right)=E_{t}[U(X, T, \omega)]
$$


and it maps $L_{T}^{b b}$ in $L_{t}^{b b}$. The CCE is easily recognized to be a CPM. From the point of view of the preferences, it offers a different numerical representation on $L_{T}^{b b}$ of the preference order induced by $E_{t}[U(\cdot, T, \omega)]$. Its economic meaning stems from its interpretation as insurance premium, called mean value principle (see Gerber [19, Chapter 5.4]). For the definition of the CCE on more general spaces, the reader is referred to Frittelli and Maggis [18]. Kupper and Schachermayer [20] have shown that the CCE process plays a considerable role in dynamic performance measures, see Section 4 for more on this topic.

Example 2.10 (Gain Loss Ratio and Best Gain Loss Ratio). Bernardo and Ledoit ([3]) introduced the static Gain-Loss Ratio as an alternative to the Sharpe Ratio in performance evaluation. Cherny and Madan [15, Section 3.2] have shown that the static Gain Loss Ratio

$$
G L R_{0}(X)=\left\{\begin{array}{cc}
\frac{E[X]}{E\left[X^{-}\right]} & \text {on } E[X]>0 \\
0 & \text { otherwise }
\end{array}\right.
$$

restricted to $L_{T}^{\infty}$ is an acceptability index in their sense, i.e. continuous from above. Note however that the very definition is slightly inconsistent within their setup, as the map is not continuous from above at 0 , since on positive, arbitrarily small payoffs it takes the value $+\infty$. It is in fact continuous from below. The conditional version of GLR is, with the convention $\frac{\xi}{0}=+\infty$ if $\xi>0$ :

$$
G L R_{t}(X)=\left\{\begin{array}{cc}
\frac{E_{t}[X]}{E_{t}\left[X^{-}\right]} & \text {on } E_{t}[X]>0 \\
0 & \text { otherwise }
\end{array}\right.
$$

$G L R_{t}$ is a CAI. It is evidently well defined on $L_{T}^{b b}, L_{t}^{b b}$-valued, monotone, nonnegative and scale invariant. Quasi concavity follows from convexity of the function $x^{-}$and linearity of (conditional) expectation exactly as in [15, Section 3.2]. Also, $\beta_{t}(+\infty)=z_{u}=+\infty$ and $z_{d}=0$. Continuity from below is easily verified, as from monotone convergence $X_{n} \in L_{T}^{b b}$, $X_{n} \uparrow X$ implies $E_{t}\left[X_{n}\right] \uparrow E_{t}[X]$ and dominated convergence gives $E_{t}\left[X_{n}^{-}\right] \downarrow E_{t}\left[X^{-}\right]$. In particular the static $G L R_{0}$ is continuous from below. Property 6 of CPMs is also satisfied, since when $z>0$

$$
A_{t}^{z} \cap L_{t}^{b b}=\left\{\xi \in L_{t}^{b b} \mid \beta_{t}(\xi) \geq z\right\}=\left\{\xi \in L_{t}^{b b}, \xi>0\right\}
$$

We only need to check locality. Note that when $B \in \mathcal{F}_{t}, P(B) \neq 0$ :

$$
\beta_{t}\left(X I_{B}\right)=\left\{\begin{array}{cc}
\frac{E_{t}[X]}{E_{t}\left[X^{-}\right]} & \text {on }\left\{E_{t}\left[X I_{B}\right]>0\right\}=B \cap\left\{E_{t}[X]>0\right\} \\
0 & \text { otherwise }
\end{array}\right.
$$

so that $\beta_{t}\left(X I_{B}\right) I_{B}=\beta_{t}(X) I_{B}$.

The Gain Loss Ratio analysis has been recently extended by Biagini and Pinar [4]. On a general probability space and in the presence of a market, they show that the market 
optimized GLR (Best GLR, BGLR) is in turn a static acceptability index as a function of the random endowment $W$ :

$$
B G L R_{0}(W)=\sup _{R \in \mathcal{R}} \frac{E[(R+W)]}{E\left[(R+W)^{-}\right]}
$$

in which $\mathcal{R}$ are the claims replicable at zero cost. The conditional version of BLGR

$$
B G L R_{t}(W)=\sup _{R \in \mathcal{R}} \frac{E_{t}[(R+W)]}{E_{t}\left[(R+W)^{-}\right]},
$$

is also a CAI.

Example 2.11 (Reward to Risk Ratio). A generalization of the GLR is the conditional reward to risk ratio, under the usual convention $\frac{\xi}{0}=+\infty$ if $\xi>0$ :

$$
\beta_{t}(X)=\left\{\begin{array}{cc}
\frac{E_{t}[U(X)]}{\sigma_{t}(X)} & \text { on }\left\{E_{t}[U(X)]>0\right\} \\
0 & \text { otherwise }
\end{array}\right.
$$

in which

- $U$ is a concave utility function, possibly stochastic as in Example 2.8, verifying $U(+\infty)>0$

- $\sigma_{t}: L_{T}^{b b} \rightarrow L_{t}^{b b}$ is a nonnegative, monotone non increasing, convex, local and continuous from below map, with $\sigma_{t}(+\infty) \in(0, \infty)$.

It can be proved, along the same lines of the GLR case, that $\beta_{t}$ is a CPM, with $z_{u}=\frac{U(+\infty)}{\sigma_{t}(+\infty)}$ and $z_{d}=0$. If $U$ and $\sigma$ are positively homogeneous, then $\beta_{t}$ is a CAI.

An example is the ratio of the conditional expectation to the conditional $p$-th lower partial moment. This has been studied by Leitner [21] in the static case, with $\sigma_{0}=$ $E\left[\left(X^{-}\right)^{p}\right]^{\frac{1}{p}}$ and fixed $p>1$. Its conditional version is thus the CAI

$$
\beta_{t}(X)=\left\{\begin{array}{cc}
\frac{E_{t}[X]}{\left(E_{t}\left[\left(X^{-}\right)^{p}\right]\right)^{\frac{1}{p}}} & \text { on }\left\{E_{t}[X]>0\right\} \\
0 & \text { otherwise }
\end{array}\right.
$$

Another class of CAIs is obtained from conditional coherent risk measures, see Bion-Nadal [8] or Detlefsen and Scandolo [16] for information on conditional risks. Fix a conditional coherent risk measure $\rho_{t}: L_{T}^{\infty} \rightarrow L_{t}^{\infty}$ and continuous from below. Then, extend it to $L_{T}^{b b}$ using the continuity from below property and finally set $\sigma_{t}:=\rho_{t} \vee 0$. To fix the ideas, $\sigma_{t}$ could be the truncation of the conditional Average Value at Risk at level $\lambda$, $\lambda \in L_{t}^{\infty}, 0<\lambda<1$, that is

$$
\rho_{t}(X)=\operatorname{ess}_{\sup } \operatorname{su}_{\left\{Q \mid Q_{\mid \mathcal{F}_{t}}=P_{\mid \mathcal{F}_{t}}, \frac{d Q}{d P} \leq \lambda^{-1}\right\}} E_{t}^{Q}[-X]
$$

The resulting CPM is

$$
\beta_{t}(X)=\left\{\begin{array}{cc}
\frac{E_{t}[U(X)]}{\rho_{t} \vee 0} & \text { on }\left\{E_{t}[U(X)]>0\right\} \\
0 & \text { otherwise }
\end{array}\right.
$$


which is a CAI when $U$ is positively homogeneous. Note that when $t=0$ and $U(x)=$ $x$, this class boils down to the RAROC class in [15, Section 3.4]. The only difference is we do not set $\beta_{t}=+\infty$ where $\rho_{t} \leq 0$. This choice in fact ignores the effect of reward on acceptable claims. Here, $\beta_{t}=+\infty$ on $\rho_{t} \leq 0, E_{t}[U(X)]>0$ and null on $\rho_{t} \leq 0, E_{t}[U(X)] \leq 0$.

Remark 2.12. In the two examples above, GLR and the more general Reward to Risk Ratio, the index is set to zero when the reward is non positive. This is not surprising, since in the end any performance measure must be optimized. Then of course the optimizer(s) (or at least the maximizing sequence) will have positive reward. For an effective analysis of generalized Sharpe Ratios, the interested reader may consult Cerný [12].

\section{Parametric families of conditional convex risk mea- sures and conditional performance measures: a one- to-one relation}

As anticipated in the Introduction, this section generalizes and completes the findings of [6. 15, 24] for acceptability indexes.

\subsection{From a CPM to the induced family of risks}

Definition 3.1. Let $z_{d}<z<z_{u}$ and consider $A_{t}^{z}=\left\{X \mid \beta_{t}(X) \geq z\right\}$. Define

$$
\rho_{t}^{z}(X):=\operatorname{essinf}\left\{\xi \in L_{t}^{b b} \mid \beta_{t}(X+\xi) \geq z\right\}=\operatorname{essinf}\left\{\xi \in L_{t}^{b b} \mid X+\xi \in A_{t}^{z}\right\}
$$

Note $\rho_{t}^{z}$ is in the form of capital requirement with initial set of acceptable position $A_{t}^{z}$. The reader unfamiliar with this notion is referred to [17, Chapter 4.1].

The next Lemma shows some properties of $\rho_{t}^{z}$ and auxiliary results. Note that if $M_{t}^{z}(X):=\left\{\xi \in L_{t}^{b b} \mid \beta_{t}(X+\xi) \geq z\right\}$, then $\rho_{t}^{z}(X)=\operatorname{essinf} M_{t}^{z}(X)$.

Lemma 3.2. Let $z_{d}<z<z_{u}$ and fix $X \in L_{T}^{b b}$. Then

1. $M_{t}^{z}(X)$ contains a bounded variable, it is a sublattice of $L_{t}^{b b}$ and is monotone in $X$, namely

$$
X \leq Y \Rightarrow M_{t}^{z}(X) \subseteq M_{t}^{z}(Y)
$$

2. $\rho_{t}^{z}(X) \in L_{t}^{b a}$;

3. there exists a sequence $\xi_{n} \downarrow \rho_{t}^{z}(X), \xi_{n} \in M_{t}^{z}(X)$;

4. $\rho_{t}^{z}$ is local: $I_{B} \rho_{t}^{z}(X)=I_{B} \rho_{t}^{z}\left(X I_{B}\right)$ for all $B \in \mathcal{F}_{t}$; 
5. $\rho_{t}^{z}$ is translation invariant on $L_{t}^{\infty}: \rho_{t}^{z}(X+\eta)=\rho_{t}^{z}(X)-\eta$ for all $\eta \in L_{t}^{\infty}$

6. $\beta_{t}(X)>z$ on $B \in \mathcal{F}_{t}$ if and only if $\rho_{t}^{z}(X)<0$ on $B$. As a consequence,

$$
\beta_{t}(X) \leq z \text { on } B \in \mathcal{F}_{t} \Leftrightarrow \rho_{t}^{z}(X) \geq 0 \text { on } B
$$

Proof. 1. $M_{t}^{z}(X)$ is monotone by monotonicity of $\beta_{t}$. In fact, $X \geq x$ for some real $x$. From (44), as $z_{d}<z<z_{u}$ there exists $\xi$ in $L_{t}^{\infty}$ such that $\beta_{t}(\xi) \geq z$; from monotonicity, $\beta_{t}(X-x+\xi) \geq z$ so that $\xi-x \in M_{t}^{z}(X)$ and $\xi-x$ is bounded. To show closure with respect to lattice properties, let $\xi, \eta$ in $M_{t}^{z}(X)$ and $A=\{\xi \leq \eta\}$. From the locality property:

$$
\beta_{t}(X+\inf (\xi, \eta))=1_{A} \beta_{t}\left(1_{A}(X+\xi)\right)+1_{A^{c}} \beta_{t}\left(1_{A^{c}}(X+\eta) \geq z\right.
$$

This proves that $M_{t}^{z}(X)$ is closed for $\wedge$. The closure for $\vee$ is trivial from the monotonicity of $\beta_{t}$. Thus it is a sublattice of $L_{t}^{b b}$.

2. The proof of the previous item shows $\rho_{t}^{z}(X) \leq \xi-x$, for some $\xi$ in $L_{t}^{\infty}$.

3. This follows from the lattice property of $M_{t}^{z}(X)$ and [22, Chapter VI.1].

4. Locality. Fix $B \in \mathcal{F}_{t}$. Suppose $\beta_{t}\left(X I_{B}+\xi^{\prime}\right) \geq z$. In particular, the inequality holds on $B$. In the same way, for any $\xi \in M_{t}^{z}(X), \beta_{t}(X+\xi) \geq z$, and the inequality holds in particular on $B^{c}$. Set $X_{1}=\left(X I_{B}+\xi^{\prime}\right), X_{2}=(X+\xi)$. Thanks to Lemma 2.6. $X_{1} I_{B}+X_{2} I_{B^{c}}=X+\left(\xi^{\prime} I_{B}+\xi I_{B^{c}}\right) \in A_{t}^{z}$. Therefore, for any $\xi^{\prime} \in M_{t}^{z}\left(X I_{B}\right)$, $\left(\xi^{\prime} I_{B}+\xi I_{B^{c}}\right) \in M_{t}^{z}(X)$ and

$$
\rho_{t}^{z}(X) I_{B} \leq\left(\xi^{\prime} I_{B}+\xi I_{B^{c}}\right) I_{B}=\xi^{\prime} I_{B}
$$

so, taking the essinf on $\xi^{\prime}, \rho_{t}^{z}(X) I_{B} \leq \rho_{t}^{z}\left(X I_{B}\right) I_{B}$. The converse inequality is analogous.

5. Translation invariance by bounded variables is an immediate consequence of the definition of $\rho_{t}^{z}$.

6. We prove the first statement.

$\Rightarrow$ Suppose $\beta_{t}(X)>z$ on $B$. Continuity from below gives $\beta_{t}\left(X-\frac{1}{n}\right) \uparrow \beta_{t}(X)$. Set $B_{n}=\left\{\omega \in B, \beta_{t}\left(X-\frac{1}{n}\right)(\omega)>z\right\}$. Corollary 2.7, locality and translation invariance by bounded variables of $\rho_{t}^{z}$ give

$$
\rho_{t}^{z}(X) I_{B_{n}} \leq-\frac{1}{n} I_{B_{n}}
$$

Thus $\rho_{t}^{z}(X)<0$ on $B_{n}$ and, passing to the limit, $\rho_{t}^{z}(X)<0$ on $B$. 
$\Leftarrow$ Suppose $\rho_{t}^{z}(X)<0$ on $B$. For all $n \in \mathbb{N}^{*}$, let $B_{n}=\left\{\rho_{t}^{z}(X)<-\frac{1}{n}\right\} \cap B$. Then $B_{n} \uparrow B$ and from translation invariance, $\rho_{t}^{z}\left(X-\frac{1}{n}\right)<0$ on $B_{n}$. From (4) there is a (bounded) $\xi$ such that $\beta_{t}(\xi) \geq z$. From translation invariance of $\rho_{t}^{z}$, $\rho_{t}^{z}(\xi+1) \leq-1<0$. Set $X^{\prime}=\left(X-\frac{1}{n}\right) I_{B_{n}}+(\xi+1) I_{B_{n}}$. Then, locality of the risk map implies $\rho_{t}^{z}\left(X^{\prime}\right)<0$. Select an approximating sequence $\xi_{k} \downarrow \rho_{t}^{z}\left(X^{\prime}\right)$ as in item 3 above. By locality and monotonicity,

$$
\beta_{t}\left(X^{\prime}\right) I_{\left\{\xi_{k}<0\right\}} \stackrel{\text { loc }}{=} \beta_{t}\left(X^{\prime} I_{\left\{\xi_{k}<0\right\}}\right) I_{\left\{\xi_{k}<0\right\}} \geq \beta_{t}\left(\left(X^{\prime}+\xi_{k}\right) I_{\left\{\xi_{k}<0\right\}}\right) I_{\left\{\xi_{k}<0\right\}} \geq z I_{\left\{\xi_{k}<0\right\}}
$$

and, sending $k$ to infinity, we get $\beta_{t}\left(X^{\prime}\right) \geq z$. Property 3 -b) of CPMs (strict monotonicity) ensures $\beta_{t}\left(X^{\prime}+\frac{1}{n}\right)>\beta_{t}\left(X^{\prime}\right)$ on $\left\{\beta_{t}\left(X^{\prime}\right)<z_{u}\right\} \cap\left\{\beta_{t}\left(X^{\prime}+\frac{1}{n}\right)>\right.$ $\left.z_{d}\right\}$. The locality property of $\beta_{t}$ and $z>z_{d}$ gives then $\beta_{t}(X)=\beta_{t}\left(X^{\prime}+\frac{1}{n}\right)>z$ on $B_{n} \cap\left\{\beta_{t}\left(X^{\prime}\right)<z_{u}\right\}$. This result is satisfied for all $n$, and since on $\left\{\beta_{t}\left(X^{\prime}\right)=\right.$ $z_{u}$ \} by monotonicity $\beta_{t}(X)=z_{u}>z$, then $\beta_{t}(X)>z$ on $B$.

To show (6), suppose $\rho_{t}^{z}(X) \geq 0$ on $B$. Let $A=\left\{\beta_{t}(X)>z\right\} \cap B \in \mathcal{F}_{t}$. On $A$, $\rho_{t}^{z}(X)<0$. It follows that $P(A)=0$. Thus $\beta_{t}(X) \leq z$ on $B$. The proof of the other implication is similar.

Proposition 3.3. $\quad$ i) For fixed $z_{u}>z>z_{d}$, consider the map $\rho_{t}^{z}$. Then, $\rho_{t}^{z}: L_{T}^{b b} \rightarrow L_{t}^{b a}$ and is

1. $L_{t}^{\infty}$-valued if restricted to $E_{T}^{\infty}$,

2. convex;

3. monotone non increasing, and it can be calculated as:

$$
\rho_{t}^{z}(X)=\operatorname{essinf}\left\{\xi \in L_{t}^{\infty} \mid X+\xi \in A_{t}^{z}\right\}
$$

4. local, $I_{B} \rho_{t}^{z}(X)=I_{B} \rho_{t}^{z}\left(X I_{B}\right)$ for all $B \in \mathcal{F}_{t}$

5. translation invariant on $L_{t}^{b b}: \rho_{t}^{z}(X+\xi)=\rho_{t}^{z}(X)-\xi$, for all $\xi \in L_{t}^{b b}$;

6. continuous from below:

$$
X_{n} \uparrow X \Rightarrow \rho_{t}^{z}\left(X_{n}\right) \downarrow \rho_{t}^{z}(X)
$$

and is therefore a conditional convex risk measure on $L_{T}^{b b}$, continuous from below.

7. If $\beta_{t}$ is scale invariant (in particular, when $\beta_{t}$ is a CAI), $\rho_{t}^{z}$ is a conditional coherent risk measure on $L_{T}^{b b}$.

ii) Moreover, for any fixed $X \in L_{T}^{b b}$, the process $\rho_{t}^{z}(X)_{z_{d}<z<z_{u}}$ is a non decreasing and continuous function of $z \in\left(z_{d}, z_{u}\right)$.

In case $z_{d}=-\infty$,

$$
\lim _{z \downarrow-\infty} \operatorname{ess} \sup \rho_{t}^{z}(0)=-\infty
$$


Proof. i) By Lemma 3.2 item 2, $\rho_{t}^{z}$ is $L_{t}^{b a}$-valued.

1. Bounded on bounded positions. Suppose $X \in \mathrm{七}_{T}^{\infty}$. From Lemma $3.2, M_{t}^{z}\left(\|X\|_{\infty}\right) \supseteq$ $M_{t}^{z}(X)$. So, $\rho_{t}^{z}\left(\|X\|_{\infty}\right) \leq \rho_{t}^{z}(X)$. Now, $\|X\|_{\infty}+M_{t}^{z}\left(\|X\|_{\infty}\right) \subseteq A_{t}^{z} \cap L_{t}^{b b}$. Since $z>z_{d}$, Property 6 of CPMs ensures that $A_{t}^{z} \cap L_{t}^{b b}$ has a uniform lower bound $x_{z}$. Therefore

$$
x_{z}-\|X\|_{\infty} \leq \operatorname{essinf} M_{t}^{z}\left(\|X\|_{\infty}\right)=\rho_{t}^{z}\left(\|X\|_{\infty}\right) \leq \rho_{t}^{z}(X)
$$

2. Convexity. Fix $X, Y$ and take a convex combination $Z=c X+(1-c) Y$ with $c \in[0,1]$. For any $\xi \in M_{t}^{z}(X), \psi \in M_{t}^{z}(Y)$, then quasi concavity of $\beta_{t}$ implies

$$
\beta_{t}(Z+(c \xi+(1-c) \psi))=\beta_{t}(c(X+\xi)+(1-c)(Y+\psi)) \geq z
$$

that is $\rho_{t}^{z}(Z) \leq c \xi+(1-c) \psi$. Taking the essential infimum over $\xi$ and $\psi$, $\rho_{t}^{z}(Z) \leq c \rho_{t}^{z}(X)+(1-c) \rho_{t}^{z}(Y)$.

3. Monotonicity (in the opposite direction) follows easily from monotonicity of $\beta_{t}$. Since $M_{t}^{z}(X)$ contains a bounded variable $\xi^{*}$ and is a sublattice of $L_{t}^{b b}$ by item 1, Lemma 3.2, $M^{\prime}:=\left\{\xi \wedge \xi^{*} \mid \xi \in M_{t}^{z}(X)\right\} \subseteq M_{t}^{z}(X) \cap L_{t}^{\infty}$ and $\rho_{t}^{z}(X)=\operatorname{essinf} M_{t}^{z}(X)=\operatorname{essinf} M^{\prime}$.

4. Locality is proved in Lemma 3.2, item 4.

5. A combination of localization and translation invariance over $L_{t}^{\infty}$ gives translation invariance for all $\xi \in L_{t}^{b b}$. In fact, suppose first $\xi<+\infty$. Then,

$\left(\rho_{t}^{z}(X)-\xi\right) I_{\{\xi \leq n\}}=\left(\rho_{t}^{z}(X)-\xi \wedge n\right) I_{\{\xi \leq n\}}=\rho_{t}^{z}(X+\xi \wedge n) I_{\{\xi \leq n\}}=\rho_{t}^{z}(X+\xi) I_{\{\xi \leq n\}}$

for all $n$ and the result follows by letting $n \uparrow \infty$. If $B:=\{\xi=+\infty\}$ has positive probability, then

$$
\rho_{t}^{z}(X+\xi) I_{B}=\rho_{t}^{z}(+\infty) I_{B}=(-\infty) I_{B}
$$

where the second equality (convention $0 \cdot \infty=0$ ) follows from $M_{t}^{z}(+\infty)=L_{t}^{b b}$. Therefore for any $\xi \in L_{t}^{b b}$

$\rho_{t}^{z}(X+\xi)=\rho_{t}^{z}(X+\xi) I_{B^{c}}+\rho_{t}^{z}(X+\xi) I_{B}=\left(\rho_{t}^{z}(X)-\xi\right) I_{B^{c}}+(-\infty) I_{B}=\rho_{t}^{z}(X)-\xi$

since $\rho_{t}^{z}(X)$ is bounded above and thus on $B \rho_{t}^{z}(X)-\xi=-\infty$.

6. Continuity from below. Let $X_{n} \uparrow X$ and suppose the set $C=\left\{\rho_{t}^{z}(X)<\right.$ $\left.\lim _{n} \rho_{t}^{z}\left(X_{n}\right)\right\}$ has positive probability. For $q \in \mathbb{Q}$, let $C_{q}=\left\{\rho_{t}^{z}(X)<q \leq\right.$ $\left.\lim _{n} \rho_{t}^{z}\left(X_{n}\right)\right\}$. Since $C=\bigcup_{q \in \mathbb{Q}} C_{q}, C_{q}$ has positive probability for some $q$. By translation invariance and Lemma 3.2 item 6 , such chain has two implications: $\beta_{t}(X+q)>z$ on $C_{q}$, and $\beta_{t}\left(X_{n}+q\right) \leq z$ on $C_{q}$. From the continuity from below of $\beta_{t}$ the last inequality implies that $\beta_{t}(X+q) \leq z$ on $C_{q}$, a contradiction. 
7. when $\beta_{t}$ is scale invariant for any $k>0$

$$
\rho_{t}^{z}(k X)=\operatorname{essinf}\left\{\xi \mid \beta_{t}(k X+\xi) \geq z\right\}=k \operatorname{essinf}\left\{\xi^{\prime} \mid \beta_{t}\left(X+\xi^{\prime}\right) \geq z\right\}=k \rho_{t}^{z}(X)
$$

where the second equality holds thanks to scale invariance of $\beta_{t}$.

ii) The process $\rho_{t}^{z}(X)_{z_{d}<z<z_{u}}$ is non decreasing in $z$ as a simple consequence of monotonicity of $\beta_{t}$. In particular for any fixed $X$, any fixed $z^{*} \in\left(z_{d}, z_{u}\right), \rho_{t}^{z}(X)$ has a left limit and a right limit in $z^{*}$. Assume that $\lim _{z \uparrow z^{*}} \rho_{t}^{z}(X) \neq \lim _{z \downarrow z^{*}} \rho_{t}^{z}(X)$. Then there are $p<q$ in $\mathbb{R}$ such that $A_{p, q}=\left\{\lim _{z \uparrow z^{*}} \rho_{t}^{z}(X)<p<q \leq \lim _{z \downarrow z^{*}} \rho_{t}^{z}(X)\right\}$ has positive probability. By Lemma 3.2, item 6, and translation invariance, for all $u<z^{*}, \beta_{t}(X+p)>u$ and for all $v>z^{*}, \beta_{t}(X+q) \leq v$ on $A_{p, q}$. Passing to the limit it follows from the monotonicity of $\beta_{t}$ that $\beta_{t}(X+p)=\beta_{t}(X+q)=z^{*}$ on $A_{p, q}$. This is in contradiction with the strict monotonicity of CPM on constant positive shifts, property 3.(b) in Definition 2.1.

To conclude the proof, the $z$-monotonicity of $\rho_{t}^{z}(0)$ implies the existence of the limit $l:=\lim _{z \downarrow-\infty} \operatorname{ess} \sup \rho_{t}^{z}(0)$. For any $n \in \mathbb{N}$ there is $z_{n}$ such that $\beta_{t}(-n) \geq z_{n}$ and by monotonicity of $\beta_{t}$ we may assume $z_{n}$ is non increasing. So, $\rho_{t}^{z_{n}}(0) \leq-n$ and

$$
\lim _{z \downarrow-\infty} \operatorname{ess} \sup \rho_{t}^{z}(0) \leq \lim _{n \rightarrow+\infty} \operatorname{ess} \sup \rho_{t}^{z_{n}}(0)=-\infty
$$

Remark 3.4. The inclusion $A_{t}^{z} \subseteq\left\{\rho_{t}^{z} \leq 0\right\}$ is always satisfied by definition of $\rho_{t}^{z}$, but may be strict due to lack of order upper semicontinuity. So in general $A_{t}^{z}$ is only a subset of the acceptance set of $\rho_{t}^{z}$. For example, pick any static $(t=0)$ CAI unbounded above and null over negative variables, e.g. the GLR from Example 2.10. Then, for any $z>0$

$$
\rho_{0}^{z}(0)=\operatorname{essinf}\left\{c \mid \beta_{0}(c) \geq z\right\}=0
$$

Indeed $\beta_{0}\left(\frac{1}{n}\right)=+\infty$ from Corollary 2.4 and thus $\frac{1}{n} \in A_{0}^{z}$ for every $n$. But $\beta_{0}(0)=0$ so that $0 \in\left\{\rho_{0}^{z} \leq 0\right\} \backslash A_{0}^{z}$.

Remark 3.5. Continuity from below for the risk $\rho_{t}^{z}$, which is monotone non increasing, is equivalent to order upper semicontinuity wrt sequences uniformly bounded from below:

$$
X_{n} \geq c,\left|X_{n}-X\right| \leq Y_{n} \downarrow 0 \Rightarrow \rho_{t}^{z}(X) \geq \limsup _{n} \rho_{t}^{z}\left(X_{n}\right)
$$

The proof, mutatis mutandis, is identical to that of Lemma 2.2 .

Corollary 3.6. For all $z \in\left(z_{d}, z_{u}\right)$, the map $\rho_{t}^{z}$ admits the following dual representation on $L_{T}^{\infty}$

$$
\rho_{t}^{z}(X)=\operatorname{ess} \max _{Q \in \mathcal{Q}}\left(E_{t}^{Q}[-X]-\alpha_{t}(Q)\right)
$$


where: $\mathcal{Q}$ is the set of probability measures on $\left(\Omega, \mathcal{F}_{T}\right)$ absolutely continuous wrt $P$ and such that $\left.Q\right|_{\mathcal{F}_{t}}=\left.P\right|_{\mathcal{F}_{t}}$; and $\alpha_{t}$ is the penalty function

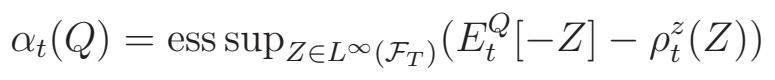

Therefore, the restriction of the risk measure to $L_{T}^{\infty}$ is continuous from above. Moreover, for all $X$ in $L_{T}^{b b}$,

$$
\rho_{t}^{z}(X)=\lim _{n \rightarrow \infty}\left(E_{t}^{Q_{n}}[-(X \wedge n)]-\alpha_{t}\left(Q_{n}\right)\right)
$$

for a suitable sequence $Q_{n} \in \mathcal{Q}$.

Proof. The first assertion is the well known representation result for conditional risk measures continuous from below, see Bion-Nadal [8, 9]. It is well known that the representation (9) implies continuity from above of the restriction of the risk measure to $L_{T}^{\infty}$. Finally, since $\rho_{t}^{z}(X)=\lim _{n} \rho_{t}^{z}(X \wedge n)$ (10) follows directly from (9) and continuity from below.

Definition 3.7. The family $\left(\rho_{t}^{z}\right)_{z_{d}<z<z_{u}}$ is called the family of risk measures induced by the CPM $\beta_{t}$.

Example 3.8. The family induced by conditional exponential utility with random risk aversion, $\beta_{t}(X)=E_{t}\left[1-e^{-\lambda_{t} X}\right]$ as in Example [2.8, item 4, is perhaps the simplest example of all. It is a family of conditional entropic risk measures where the parameter $z$ gives only a translation effect. In fact, fix the risk aversion $\lambda_{t} \in L_{t}^{\infty}, \lambda_{t}$ positive and bounded away from zero. Then, $z_{d}=-\infty, z_{u}=1$ and, for any $z \in\left(z_{d}, z_{u}\right), \rho_{t}^{z}(X)=$ $\operatorname{essinf}\left\{\xi \mid E_{t}\left[1-e^{-\lambda_{t}(X+\xi)}\right] \geq z\right\}$. Explicitly:

$$
\rho_{t}^{z}(X)=\frac{\ln E_{t}\left[e^{-\lambda_{t} X}\right]}{\lambda_{t}}-\frac{\ln (1-z)}{\lambda_{t}}
$$

Note $\rho_{t}^{z}(0)=-\frac{\ln (1-z)}{\lambda_{t}}$, so these risk measures are not normalized. For the level $z=0$ we recover the normalized entropic risk measure.

Knowing the whole family of risks induced by a CPM amounts to the knowledge of the CPM itself.

Proposition 3.9. The CPM $\beta_{t}$ can be reconstructed from the induced family of risks $\left(\rho_{t}^{z}\right)_{z_{d}<z<z_{u}}$. In fact, suppose first $z_{d} \in \mathbb{R}$ and set $B_{X}:=\left\{\beta_{t}(X)=z_{d}\right\}$. Then

1. $B_{X}=\cap_{z \in\left(z_{d}, z_{u}\right)}\left\{\rho_{t}^{z}(X) \geq 0\right\}$

2. with the convention $0 \cdot \infty=0, \beta_{t}(X)=z_{d} I_{B_{X}}+I_{B_{X}^{c}} \xi$, where

$$
\xi:=\operatorname{ess} \sup \left\{\varphi \in \mathcal{S}_{t} \mid z_{d}<\varphi<z_{u}, \varphi=\sum_{i=1}^{n} z_{i} I_{B_{i}}, \rho_{t}^{z_{i}}(X)<0 \text { on } B_{i} \cap B_{X}^{c} \text { for all } i=1 \ldots n\right\}
$$


In case $z_{d}=-\infty$, we get the simplified relation

$$
\beta_{t}(X)=\operatorname{ess} \sup \left\{\varphi \in \mathcal{S}_{t} \mid \varphi<z_{u}, \varphi=\sum_{i=1}^{n} z_{i} I_{B_{i}}, \rho_{t}^{z_{i}}(X)<0 \text { on } B_{i} \text { for all } i=1 \ldots n\right\} .
$$

Proof. Item 1 is a consequence of ([6) . To prove item 2, note that by localization we can assume $\beta_{t}(X)>z_{d}$ everywhere. The following proof will then hold also in case $z_{d}=-\infty$. Note

$$
\begin{aligned}
\beta_{t}(X) & =\operatorname{ess} \sup \left\{\varphi \in \mathcal{S}_{t} \mid \varphi<\beta_{t}(X)\right\} \\
& =\operatorname{ess} \sup \left\{\varphi \in \mathcal{S}_{t} \mid z_{d}<\varphi<z_{u}, \varphi=\sum_{i=1}^{n} z_{i} I_{B_{i}}, \beta_{t}(X)>z_{i} \text { on } B_{i} \text { for all } i=1 \ldots n\right\} \\
& =\operatorname{ess} \sup \left\{\varphi \in \mathcal{S}_{t} \mid z_{d}<\varphi<z_{u}, \varphi=\sum_{i=1}^{n} z_{i} I_{B_{i}}, \rho_{t}^{z_{i}}(X)<0 \text { on } B_{i} \text { for all } i=1 \ldots n\right\} \\
& =\beta_{t}(X)
\end{aligned}
$$

in which the first two equalities give simply the approximation of a bounded from below claim by bounded variables, while the last two hold thanks to Lemma [3.2, item 6 first part.

\subsection{From a parametric family of risks to the induced CPM}

A one-parameter family of conditional risk measures $\left(\sigma_{t}^{z}\right)_{z}$ which satisfies certain regularity assumptions induces a CPM. The idea is to take the relations in items 1, 2 of Proposition 3.9 as definitions of a map $\beta_{t}$ and to show it is indeed a CPM.

Definition 3.10 (Standard family of risk measures). Fix a non empty open interval $I=\left(z_{d}, z_{u}\right) \subseteq \mathbb{R}$, possibly unbounded. Let $\left(\sigma_{t}^{z}\right)_{z \in I}$ be a parametric family of conditional convex risk measures continuous from below on $L_{T}^{b b}$, non decreasing and continuous in the parameter $z$, i.e. a collection of maps satisfying the conditions:

a) For fixed $z \in I, \sigma_{t}^{z}: L_{T}^{b b} \rightarrow L_{t}^{b a}$ is:

1. $L_{t}^{\infty}$-valued when restricted to $L_{T}^{\infty}$;

2. convex, i.e. $\forall c \in[0,1], \forall X, Y \in L_{T}^{b b}, \sigma_{t}^{z}(c X+(1-c) Y) \leq c \sigma_{t}^{z}(X)+(1-c) \sigma_{t}^{z}(Y)$;

3. monotone non increasing

4. translation invariant on $L_{t}^{b b}: \sigma_{t}^{z}(X+\xi)=\sigma_{t}^{z}(X)-\xi$ whenever $\xi \in L_{t}^{b b}$;

5. local: $\sigma_{t}^{z}(X) I_{B}=\sigma_{t}^{z}\left(X I_{B}\right) I_{B}$ for all $B \in \mathcal{F}_{t}$;

6. continuous from below: $X_{n} \uparrow X \Rightarrow \sigma_{t}^{z}\left(X_{n}\right) \downarrow \sigma_{t}^{z}(X)$.

b) For fixed $X \in L_{T}^{b b}$, the paths of the process $\left(\sigma_{t}^{z}(X)\right)_{z}$ are almost surely non decreasing and continuous on $I$. 
c) If $z_{d}=-\infty$, then $\lim _{z \rightarrow-\infty} \operatorname{ess} \sup \sigma_{t}^{z}(0)=-\infty$.

Then, the family of risk measures $\left(\sigma_{t}^{z}\right)_{z \in I}$ is called standard.

Proposition 3.11. Let $\left(\sigma_{t}^{z}\right)_{z \in I}$ be a standard family of conditional risk measures. Then, with the convention $0 \cdot \infty=0$ define on $L_{T}^{b b}$ the map $\beta_{t}$ as

$\beta_{t}(X):=z_{d} I_{B_{X}}+I_{B_{X}^{c}} \operatorname{ess} \sup \left\{\varphi \in \mathcal{S}_{t} \mid z_{d}<\varphi<z_{u}, \varphi=\sum_{1}^{n} z_{i} I_{B_{i}}, \sigma_{t}^{z_{i}}(X)<0\right.$ on $\left.B_{i} \cap B_{X}^{c}\right\}$

in which $B_{X}:=\cap_{z \in I}\left\{\sigma_{t}^{z}(X) \geq 0\right\}$. The map $\beta_{t}$ is a CPM, called the CPM generated $\boldsymbol{b} \boldsymbol{y}\left(\sigma_{t}^{z}\right)_{z \in I}$, and the thresholds $z_{d}, z_{u}$ are the essential infimum and supremum of $\beta_{t}$. If $\left(\sigma_{t}^{z}\right)_{z \in I}$ is a family of conditional coherent (i.e. sublinear) risk measures and if $z_{d}=0, \beta_{t}$ is a $C A I$.

Proof. The proof is split in a number of steps.

1. $\beta_{t}: L_{T}^{b b} \rightarrow L_{t}^{b b}$.

Fix $X \in L_{T}^{b b}$. When $z_{d}$ is finite, by definition $\left\{\beta_{t}(X)=z_{d}\right\} \equiv B_{X}$. So, if $z_{d}$ is finite we are done. In case $z_{d}=-\infty$, by property c) of standard families, there exists $z^{*} \in \mathbb{R}$ such that $\sigma_{t}^{z^{*}}(0)<-\left\|X^{-}\right\|_{\infty}$ and by monotonicity and translation invariance $\sigma_{t}^{z^{*}}(X) \leq \sigma_{t}^{z^{*}}\left(-\left\|X^{-}\right\|_{\infty}\right)<0$, from which $\beta_{t}(X) \geq z^{*}$.

2. Locality.

$$
\begin{aligned}
\beta_{t}\left(X I_{B}\right) I_{B}= & z_{d} I_{B_{X} \cap B}+ \\
& I_{B_{X}^{c} \cap B} \operatorname{ess} \sup \left\{\varphi=\sum_{1}^{n} z_{i} I_{B_{i}}, z_{i} \in I, \sigma_{t}^{z_{i}}\left(X I_{B}\right)<0 \text { on } B_{i} \cap B_{X}^{c}\right\} \\
= & I_{B}\left(z_{d} I_{B_{X}}+I_{B_{X}^{c}} \operatorname{ess} \sup \left\{\varphi=\sum_{1}^{n} z_{i} I_{B_{i}}, z_{i} \in I, \sigma_{t}^{z_{i}}(X)<0 \text { on }\left(B_{i} \cap B\right) \cap B_{X}^{c}\right\}\right.
\end{aligned}
$$

where the second equality follows from locality of $\sigma_{t}^{z_{i}}$. When $\left\{B_{1}, \ldots B_{n}\right\}$ varies among all finite $\mathcal{F}_{t}$-measurable partitions of $\Omega,\left\{B_{1} \cap B, B_{1} \cap B^{c}, \ldots, B_{n} \cap B, B_{n} \cap B^{c}\right\}$ still varies among all such partitions and therefore we get that the last term displayed above equals $I_{B} \beta_{t}(X)$.

3. If $z_{u}>z>z_{d}$, then $\beta_{t}(X)>z$ on $B$ if and only if $\sigma_{t}^{z}(X)<0$ on $B$. The arrow $(\Rightarrow)$ is a consequence of the definition (11). Conversely, suppose $\sigma_{t}^{z}(X)<0$ on $B$. By the $z$-(right) continuity and monotonicity of the process $\sigma_{t}^{z}(X)_{z}$ we have

$$
\left\{\sigma_{t}^{z}(X)<0\right\}=\cup_{n \in \mathbb{N}^{*}, z+\frac{1}{n}<z_{u}}\left\{\sigma_{t}^{z+\frac{1}{n}}(X)<0\right\}
$$

so that $B=\cup_{n \in \mathbb{N}^{*}, z+\frac{1}{n}<z_{u}} B_{n}$, where $B_{n}:=B \cap\left\{\sigma_{t}^{z+\frac{1}{n}}(X)<0\right\}$. But on $B_{n}$, by definition of $\beta_{t}$ and by its locality, $\beta_{t}(X) \geq z+\frac{1}{n}>z$. 
4. Quasi concavity. By locality, it is enough to show that, for any $z_{u}>z>z_{d}$, if $\beta_{t}(X)>z$ and $\beta_{t}(Y)>z$, then $\beta_{t}(c X+(1-c) Y)>z$. In virtue of item 3 above, this is equivalent to $\sigma_{t}^{z}(X)<0, \sigma_{t}^{z}(Y)<0$ imply $\sigma_{t}^{z}(c X+(1-c) Y)<0$, which holds by convexity of $\sigma_{t}^{z}$.

5. Thresholds. Let $z \in I$. Since $\sigma_{t}^{z}(0) \in L_{t}^{\infty}, \sigma_{t}^{z}(0) \leq b_{z}$ for some constant. Therefore, for all $n \sigma_{t}^{z}(n) \leq b_{z}-n$, and thus from decreasing monotonicity, $\sigma_{t}^{z}(+\infty)=-\infty$ for all $z$. This implies $\beta_{t}(+\infty)=z_{u}$. To show $z_{d}$ is the lower threshold, set

$$
\lim _{n} \beta_{t}(-n):=\eta
$$

The limit exists as $\beta_{t}$ is clearly monotone non decreasing. The set $\left\{\eta>z_{d}\right\}$ has zero probability. In fact,

$$
\left\{\eta>z_{d}\right\}=\cup_{z_{u}>q>z_{d}, q \in \mathbb{Q}}\{\eta>q\}
$$

On every $\{\eta>q\} \beta_{t}(-n)>q$ for all $n$ and this implies $\sigma_{t}^{q}(0)<-n$ for all $n$, which leads to a contradiction unless $P(\eta>q)=0$.

6. Non decreasing monotonicity is clear, so we just show strict monotonicity over constant positive shifts. Let $c>0$. Assume that $\beta_{t}(X)<z_{u}, \beta_{t}(X+c)>z_{d}$. Set $B=\left\{\beta_{t}(X+c)=\beta_{t}(X)\right\}$ and let us prove $P(B)=0$. On $B$, for any $z_{u}>z>z_{d}$ such that $\beta_{t}(X+c)>z$, also $\beta_{t}(X)>z$. Reformulating thanks to item 3 above, for any such $z, \sigma_{t}^{z}(X+c)<0$ on $B$ implies $\sigma_{t}^{z}(X)<0$ on $B$. Thus on $B$ the image of the path of $\sigma_{t}^{z}(X)_{z}$ does not contain $[0, c)$. Due to the continuity of the map $z \rightarrow \sigma_{t}^{z}(X)$ it follows that on $B, \sigma_{t}^{z}(X)<0$ for all $z$ or that $\sigma_{t}^{z}(X) \geq c$ for all $z \in\left(z_{d}, z_{u}\right)$. The first condition means $\beta_{t}(X)=z_{u}$ and the second $\beta_{t}(X+c)=z_{d}$, a contradiction unless $P(B)=0$.

7. Continuity from below. Let $X_{n} \uparrow X$. Then, from monotonicity of $\beta_{t}, \beta_{t}(X) \geq$ $\lim _{n} \beta_{t}\left(X_{n}\right)$. We obtain an equality as soon as if for some $z_{u}>z>z_{d} \beta_{t}(X)>z$ on $B$, then $\lim _{n} \beta_{t}\left(X_{n}\right)>z$ on $B$. By locality, since $\beta_{t}\left(X I_{B}\right) I_{B}=\beta_{t}(X) I_{B}$, by eventually replacing $X$ with $X I_{B}$ and $X_{n}$ with $X_{n} I_{B}$, we can assume $B=\Omega$. By item 3 above, $\sigma_{t}^{z}(X)<0$. From continuity from below of the risk measure $\sigma_{t}^{z}$, $\sigma_{t}^{z}\left(X_{n}\right) \downarrow \sigma_{t}^{z}(X)$. If $C_{n}:=\left\{\sigma_{t}^{z}\left(X_{n}\right)<0\right\}$, then $C_{n} \uparrow \Omega$ and by definition of $\beta_{t}$

$$
\lim _{n} \beta_{t}\left(X_{n}\right) \geq \beta_{t}\left(X_{n}\right)>z \text { on } C_{n}
$$

whence $\lim _{n} \beta_{t}\left(X_{n}\right)>z$.

8. Property 6 of CPM. If $z_{d}<z<z_{u}$ and if $z-\epsilon>z_{d}$, then $\left\{\beta_{t} \geq z\right\} \subseteq\left\{\beta_{t}>z-\epsilon\right\}=$ $\left\{\sigma_{t}^{z-\epsilon}<0\right\}$ by monotonicity and item 3. So,

$$
\xi \in L_{t}^{b b}, \xi \in A_{t}^{z} \Rightarrow \sigma_{t}^{z-\epsilon}(\xi)<0 \Rightarrow \sigma_{t}^{z-\epsilon}(0)<\xi
$$

and since $\sigma_{t}^{z-\epsilon}(0) \in L_{t}^{\infty}$ we conclude $A_{t}^{z} \cap L_{t}^{b b}$ is uniformly bounded from below. 
9. Properties 7 and 8 for CAIs are easily verified in case $\left(\sigma_{t}^{z}\right)_{z \in I}$ is a family of conditional coherent risk measures and $z_{d}=0$.

\subsection{A one to one relation between standard families of risks and CPMs}

Proposition 3.12. We tie together the results shown so far:

1. a CPM $\beta_{t}$ induces a standard family of convex risk measures $\left(\rho_{t}^{z}\right)_{z_{d}<z<z_{u}}$; if $\beta_{t}$ is a CAI, the risks are coherent;

2. a standard family of convex risk measures generates a CPM; if in addition the risks are coherent, they induce a CAI;

3. $\left(\rho_{t}^{z}\right)_{z_{d}<z<z_{u}}$ is the unique standard family of convex risk measures generating the $C P M \beta_{t}$, in the sense that any other standard family $\left(\sigma_{t}^{z}\right)_{z_{d}<z<z_{u}}$ generating $\beta_{t}$ satisfies

$$
\rho_{t}^{z}(X)=\sigma_{t}^{z}(X)
$$

Proof. 1. The family $\left(\rho_{t}^{z}\right)_{z}$ is standard from Proposition 3.3 .

2. This is Proposition 3.11,

3. If $\left(\rho_{t}^{z}\right)_{z}$ is the family induced by $\beta_{t}$, then the family in turn generates a CPM which by Proposition 3.9 is exactly $\beta_{t}$. If, for any other standard family $\left(\sigma_{t}^{z}\right)_{z_{d}<z<z_{u}}$, we show that the equality $\left\{\sigma_{t}^{z} \geq 0\right\}=\left\{\rho_{t}^{z} \geq 0\right\}$ holds, then the uniqueness of $\left(\rho_{t}^{z}\right)_{z}$ will follow from translation invariance. Fix then $X \in L_{T}^{b b}$. From (6) and from the proof Proposition 3.11, item 3,

$$
\rho_{t}^{z}(X) \geq 0 \text { iff } \beta_{t}(X) \leq z \text { iff } \sigma_{t}^{z}(X) \geq 0
$$

Remark 3.13. If $\mathcal{F}_{t}$ is trivial and if we are in the scale invariant case, the above Proposition becomes a static result which can be rephrased as follows:

$A \operatorname{map} \beta: L_{T}^{b b} \rightarrow[0,+\infty]$ is an acceptability index if and only if there exists a unique standard family of coherent risk measures $\left(\sigma^{z}\right)_{z \in\left(0, z_{u}\right)}$ such that

$$
\beta(X)=\sup \left\{z \mid \sigma^{z}(X)<0\right\}
$$

with the convention $\sup \emptyset=0$. The standard family is $\left(\rho^{z}\right)_{z \in\left(0, z_{u}\right)}$, with $\rho^{z}(X)=\operatorname{essinf}\{c \mid \beta(X+c) \geq z\}$. 
This is the ( $L_{T}^{b b}$-formulation of the) result in [15], Theorem 1 and Proposition 2 eqq. (4) and (5). Here the authors obtain a very close expression for $\beta$ as $\beta(X)=\sup \{z \mid$ $\left.\sigma^{z}(X) \leq 0\right\}$, with non strict inequality, and they get a maximal standard family, possibly non unique. Such difference is due to the different order continuity assumption of the map, from below and not from above, and to our assumption of strict monotonicity over constant positive shifts. Our result also generalizes to the conditional case some of the findings of Drapeau and Kupper for static quasi-convex risk measures [13, Theorem 1.7].

\section{Dynamic Performance Measures}

\subsection{Definition and time consistency}

Definition 4.1. A Dynamic Performance Measure (DPM) is a family $\beta=\left(\beta_{t}\right)_{t \in \mathcal{T}}$ of CPMs with the properties:

1. $\beta_{t}: L_{T}^{b b} \rightarrow L_{t}^{b b}$

2. the (essential) supremum and infimum $z_{u}, z_{d}$ do not depend on $t \in \mathcal{T}$. 1

In case the CPMs are in particular CAIs, we call the DPM a Dynamic Acceptability Index (DAI).

The consistency across time of a DPM certainly is a key issue. The notion we choose ensures that if a future performance measure $\beta_{t}$ assesses a value to a payoff $X$ uniformly strictly higher than a certain level, the today's performance measure $\beta_{s}$ preserves such level.

Definition 4.2. A DPM (or DAI) $\beta$ is time consistent if for all $z_{d}<z<z_{u}, X \in L_{T}^{b b}$, $s, t \in \mathcal{T}, s<t$

$$
\beta_{t}(X)>z \Rightarrow \beta_{s}(X)>z,
$$

Remark 4.3. Locality of each component of the DPM $\beta$ implies that the time consistency definition can be given in the equivalent form

$$
X \in L_{T}^{b b}, B \in \mathcal{F}_{s}, \beta_{t}(X)>z \text { on } B \Rightarrow \beta_{s}(X)>z \text { on } B
$$

The simple proof relies on Corollary 2.7. A very close notion of time consistency has been given in Rosazza and Sgarra 24] with non strict inequalities in the above displayed relation, for the case of DAIs continuous from above originating from $g$-expectations defined on bounded variables.

\footnotetext{
${ }^{1}$ From a mathematical viewpoint, we could relax the requirement to $z_{u}=z_{u}(t)$ is non increasing, $z_{d}=z_{d}(t)$ is non decreasing with no substantial changes.
} 
Proposition 4.4. For a DPM $\beta$ the following are equivalent:

1. $\beta$ is time consistent;

2. for all $z_{d}<z<z_{u}, s<t,\left\{\rho_{t}^{z}<0\right\} \subseteq\left\{\rho_{s}^{z}<0\right\}$;

3. for all $z_{d}<z<z_{u} s<t,\left\{\rho_{t}^{z} \leq 0\right\} \subseteq\left\{\rho_{s}^{z} \leq 0\right\}$.

Proof. $\quad$ The equivalence 1$) \Leftrightarrow 2$ ) is an easy consequence of Lemma 3.2 , item 6 .

$2) \Rightarrow 3)$ Let $s<t$ and suppose $\rho_{t}^{z}(X) \leq 0$. By translation invariance, $\rho_{t}^{z}\left(X+\frac{1}{n}\right)<0$. From property 2$), \rho_{s}^{z}\left(X+\frac{1}{n}\right)<0$. By translation invariance, passing to the limit we get $\rho_{s}^{z}(X) \leq 0$.

$3) \Rightarrow 2$ ) Conversely, suppose 3) holds and fix $X$ such that $\rho_{t}^{z}(X)<0$. Let $B_{n}=\left\{\rho_{t}^{z}(X)<\right.$ $\left.-\frac{1}{n}\right\}$. By translation invariance and locality $\rho_{t}^{z}\left(\left(X-\frac{1}{n}\right) I_{B_{n}}+X I_{B_{n}^{c}}\right) \leq 0$, from which $\rho_{s}^{z}\left(\left(X-\frac{1}{n}\right) I_{B_{n}}+X I_{B_{n}^{c}}\right) \leq 0$. By localization and translation invariance $\rho_{s}^{z}(X)<-\frac{1}{n}$ on $B_{n}$ and passing to the limit we conclude $\rho_{s}^{z}(X)<0$.

Remark 4.5. The equivalence of the properties 2) and 3) in the above proposition is very general. It applies as soon as the translation invariance property and the local property are satisfied. In particular it is satisfied for dynamic risk measures on $L^{p}$ spaces, $1 \leq p \leq \infty$.

Remark 4.6. In the specific case of normalized risk measures Property 3 of Proposition 4.4 is known under the name of 'weak acceptance consistency', see Acciaio and Penner [1] for a detailed overview of time consistency definitions for risks and detailed references.

Corollary 4.7. Let $z_{d}<z<z_{u}$. For $Q \ll P$, let

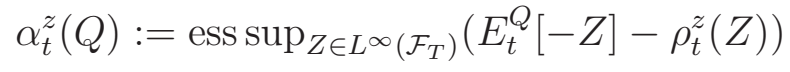

Then, if the DPM $\beta$ is time consistent, for any fixed $z$ we have

$$
\text { for all } Q \ll P, s<t, \quad E_{s}^{Q}\left[\alpha_{t}^{z}(Q)\right] \leq \alpha_{s}^{z}(Q)
$$

Proof. Acciaio and Penner [1] prove that for dynamic risk measures on $L_{T}^{\infty}$, weak time consistency is equivalent to (13). They assume the risks are normalized, but the same proof holds for the non normalized case. Therefore from Proposition 4.4, equation (13) is a necessary condition for time consistency of the DPM.

On $L_{T}^{\infty}$, the acceptance sets satisfy the following property:

Lemma 4.8. Fix $z_{d}<z<z_{u}$ and $0 \leq t<T$. Then $\left\{\rho_{t}^{z} \leq 0\right\} \cap L_{T}^{\infty}$ is weak ${ }^{*}$ closed and

$$
\overline{\left\{\rho_{t}^{z}<0\right\} \cap L_{T}^{\infty}}=\left\{\rho_{t}^{z} \leq 0\right\} \cap L_{T}^{\infty}
$$


Proof. The risk measure $\rho_{t}^{z}$ restricted to $L_{T}^{\infty}$ takes values in $L_{t}^{\infty}$. As a consequence, $\left\{\rho_{t}^{z} \leq 0\right\} \cap L_{T}^{\infty}$ is exactly the acceptance set of the risk $\rho_{t}^{z}$ restricted to $L_{T}^{\infty}$. Such set is then weak* closed because, by a standard result on conditional risk measures on $L_{T}^{\infty}[$ ], continuity from below implies continuity from above, which in turn is equivalent to the weak* closure of the acceptance set.

To prove $\left\{\rho_{t}^{z} \leq 0\right\} \cap L_{T}^{\infty}=\overline{\left\{\rho_{t}^{z}<0\right\} \cap L_{T}^{\infty}}$, it is enough to show the inclusion $\subseteq$. So, pick a bounded $Y, \rho_{t}^{z}(Y) \leq 0$ and simply consider $Y_{n}=Y+\frac{1}{n}$. Then, $\rho_{t}^{z}\left(Y_{n}\right) \leq-\frac{1}{n}$, so $Y_{n} \in\left\{\rho_{t}^{z}<0\right\}$, and $Y_{n} \rightarrow Y$ in the uniform norm topology and in particular in the weak* one.

\subsection{Time consistent DPMs: examples and counterexamples}

Example 4.9. The dynamic version of Example 2.8. Suppose we are given a stochastic dynamic utility as described at the beginning of Example 2.9. Then, $\beta$ defined as

$$
\beta_{t}(X)=E_{t}[U(X, t, \omega)]
$$

is a DPM. Thus, it is time consistent if

$$
E_{t}[U(X, t, \omega)]>z \Rightarrow E_{s}[U(X, s, \omega)]>z
$$

for any choice of dates $s<t$. Then

- if $U=U(x), \beta$ is obviously time consistent

- in the exponential case, $U(x, t, \omega)=1-e^{-\gamma_{t} x}, \beta$ is time consistent if the risk aversion process is pathwise non decreasing, i.e. $t \rightarrow \gamma_{t}(\omega)$ is non increasing.

Example 4.10 (Dynamic Certainty Equivalent). Suppose the utility $U=U(x)$ is finite on $\mathbb{R}$ and strictly increasing. Then, the dynamic certainty equivalent process $C=\left(C_{t}\right)_{t}$ :

$$
C_{t}(X)=U^{-1}\left(E_{t}[U(X)]\right)
$$

easily seen to be a time consistent DPM. It is also straightforward to prove that $C_{s}$ is normalized, i.e. $C_{s}(z)=z \forall z \in \mathbb{R}$, and satisfies the strong time consistency property: $C_{s}\left(C_{t}(X)\right)=C_{s}(X)$. Thus it is straightforward to check that the DPM $C$ is time consistent according to our definition. This example is the only possible one satisfying the law invariance, the normalization and the strong time consistency properties, as shown by Kupper and Schachermayer [20, Theorem 1.4].

Example 4.11 (Dynamic Gain-Loss Ratio). The DAI defined by

$$
G L R_{t}(X)=\left\{\begin{array}{cc}
\frac{E_{t}[X]}{E_{t}\left[X^{-}\right]} & \text {on } E_{t}[X]>0 \\
0 & \text { otherwise }
\end{array}\right.
$$


is time consistent. If $z>0$ is fixed, then $G L R_{t}(X)>z$ is equivalent to $E_{t}\left[X^{+}-(1+\right.$ $\left.z) X^{-}\right]>0$, so that, when $s<t, E_{s}\left[X^{+}-(1+z) X^{-}\right]>0$, i.e. $G L R_{s}(X)>z$. This dynamic index has been used by Bielecki et alii [7] to compute price intervals for path dependent options.

Example 4.12. As pointed out also by Bielecki et alii, [6, Example 6.5], in general the DCAIs originating from reward to risk ratio are not time consistent. The reason is that $E_{s}\left[\sigma_{t}(X)\right]$ is not in general greater than $\sigma_{s}(X)$. For instance, the reward to $p$-th lower partial moment, $1<p<+\infty$, which is but a slight variation of $G L R$ :

$$
\left\{\begin{array}{cc}
\frac{E_{t}[X]}{\left(E_{t}\left[\left(X^{-}\right)^{p}\right]\right)^{\frac{1}{p}}} & \text { on } E_{t}[X]>0 \\
0 & \text { otherwise }
\end{array}\right.
$$

is not time consistent as e.g. simple numerical examples in a two step binomial tree show.

Example 4.13 (DAI from g-expectations). In [24], the notion of dynamic acceptability index has been introduced in the particular case of a Brownian filtration from a family of $g$-expectations $\left(\mathcal{E}_{g_{\gamma}}\right)_{\gamma}$ defined on $L_{T}^{2}$. More precisely, fix a Brownian motion $B$. For every $\gamma \in \mathbb{R}_{+}$consider a driver $g_{\gamma}$ and the associated BSDE:

$$
-d Y_{t}=g_{\gamma}\left(t, Y_{t}, Z_{t}\right) d t-Z_{t} d B_{t}, \forall t \in[0, T], \text { and } Y_{T}=X
$$

with terminal condition $X \in L_{T}^{2}$. As in [24], assume $g_{\gamma}(s, \omega, z)$ is convex and uniformly Lipschitz in $z$, and $g_{\gamma}(s, \omega, 0)=0$. The (unique) solution is a couple of processes $(Y, Z)$ and $Y_{t}$ is denoted by $\mathcal{E}_{g_{\gamma}}\left(X \mid \mathcal{F}_{t}\right)$. Let $\rho_{t}^{\gamma}(X)=\mathcal{E}_{g_{\gamma}}\left(-X \mid \mathcal{F}_{t}\right)$. The family $\left(\rho_{t}^{\gamma}\right)_{t}$ is then a family of normalized dynamic risk measures continuous from above satisfying the strong time consistency property: for all $0 \leq s \leq t \leq T, \rho_{s}^{\gamma}\left(-\rho_{t}^{\gamma}(X)\right)=\rho_{s}^{\gamma}(X)$. Moreover it follows from Lemma 36.3 of Peng [23] that $\rho_{t}^{\gamma}$ defined above is continuous from below on $L_{T}^{2}$. Thanks to normalization, translation invariance and monotonicity, $\rho_{t}^{\gamma}$ is bounded on bounded variables. Therefore, any risk measure $\rho_{t}^{\gamma}$ can be uniquely extended to a normalized risk measure on $L_{T}^{b b}$, denoted in the same way, which is continuous from below and is $L_{t}^{b a}$-valued.

Suppose now that for a given $t, g_{\gamma}(t, \omega, z)$ is a continuous function of $\gamma$, uniformly in $(\omega, z)$, and is non decreasing. Then, $\left(\rho_{t}^{\gamma}\right)_{\gamma}$ is a standard family of conditional risk measures. Call $\beta_{t}(X)$ the generated CAI as given by formula (11). It is easy to check that $\beta=\left(\beta_{t}\right)_{t}$ is a DAI. It satisfies Property 3 of Proposition 4.4 thus it is time consistent.

Example 4.14 (DPM from general time consistent dynamic risk measures). Let $\left(\Omega, \mathcal{F}, \mathcal{F}_{t}, P\right)$ be a filtered probability space. For all $z_{d}<z<z_{u}$, let $\rho_{s, t}^{z}$, be a time consistent dynamic risk measure on $L^{\infty}$ i.e for all $0 \leq r \leq s \leq t \leq T, \rho_{s, t}^{z}: L_{t}^{\infty} \rightarrow L_{s}^{\infty}$ and $\rho_{r, t}^{z}(X)=\rho_{r, s}^{z}\left(-\rho_{s, t}^{z}(X)\right)$. Assume that for all $0 \leq s \leq t \leq T, \rho_{s, T}^{z}$ is continuous from below and that $\rho_{s, t}^{z}(0) \leq 0$. Assume also that $z \rightarrow \rho_{s, T}^{z}(X)$ is non decreasing and continuous for all $X$. The family $\rho_{s}^{z}=\rho_{s, T}^{z}$ is then a standard family of risks. The corresponding 
DPM is time consistent.

General examples of time consistent dynamic risk measures with possible jumps may be obtained from a stable set $\mathcal{Q}$ of probability measures all equivalent and penalties $\alpha_{s, t}(Q)$ defined for $Q \in \mathcal{Q}$ satisfying the local and cocycle properties (see Bion-Nadal [9] and [10]). For all $X \in L_{t}^{\infty}, \rho_{s t}^{z}(X)=\operatorname{ess}_{\sup _{Q \in \mathcal{Q}}}\left(E_{s}^{Q}[-X]-\alpha_{s, t}^{z}(Q)\right)$. In particular when $\mathcal{Q}$ is weakly relatively compact and the penalty is a lower semi-continuous function of $Q$ (which is the case for the minimal penalty), $\rho_{s t}^{z}$ is continuous from below. Assuming furthermore that $z \rightarrow \alpha_{s, t}^{z}(Q)$ is non increasing and continuous (uniformly in $Q$ ), the family $\rho_{s}^{z}=\rho_{s, T}^{z}$ is a standard family of risks. The families of time consistent dynamic risk measures constructed in [11] in a Markovian setting can easily be used to construct such a standard family of risks.

This construction generalizes that of DAIs from g-expectations.

\subsection{Extension of performance measures to dividend processes}

Bielecki et alii introduced in [6] a notion of dynamic acceptability index for dividend processes on a finite probability space with finite dates $\mathcal{T}$. Our goal here is to show that there is a natural correspondence between CPMs on random variables and CPMs on processes, and between DPMs on random variables and DPMs on dividend processes.

Definition 4.15. A process $D=\left(D_{t}\right)_{t \in \mathcal{T}}$ is called a (discounted) dividend process if

1. $D_{t} \in L_{t}^{b b}$, for all $t \in \mathcal{T}$

2. in case $\mathcal{T}$ is infinite, there is a discrete subset $\mathcal{T}_{D}$ of $\mathcal{T}$ such that $D_{t}=0$ if $t \notin \mathcal{T}_{D}$

3. $D_{t} \geq 0$ except for a finite number of $t \in \mathcal{T}_{D}$.

Denote by $\mathcal{D}$ the lattice of dividend processes.

Fix now $t \in \mathcal{T}$. A CPM $\beta_{t}: L_{T}^{b b} \rightarrow L_{t}^{b b}$ induces a CPM on dividend processes, $\hat{\beta}_{t}: \mathcal{D} \rightarrow L_{t}^{b b}$, by setting

$$
\left.\hat{\beta}_{t}(D):=\beta_{t}\left(\sum_{r \geq t} D_{r}\right)\right)
$$

Indeed, $\hat{\beta}_{t}$ is well defined, as $\sum_{r \geq t} D_{r}$ is bounded from below from item 3 in Definition 4.15, and $\hat{\beta}_{t}(D) \in L_{t}^{b b}$. The definition of $\hat{\beta}_{t}$ implies

$$
\hat{\beta}_{t}(D)=\hat{\beta}_{t}\left(\left(\sum_{r \geq t} D_{r}\right) I_{\{T\}}\right)
$$

where the notation $\xi I_{\{r\}}$ with $\xi \in L_{r}^{b b}$ here and in the following indicates the dividend process with a unique cashflow of $\xi$ at time $r$. So, it is straightforward to check that $\hat{\beta}_{t}$ satisfies the following properties: 
1. independence of the past and locality: for $D, D^{\prime} \in \mathcal{D}$, if for some $B \in \mathcal{F}_{t} D_{r} I_{B}=$ $D_{r}^{\prime} I_{B}$ for all $r \geq t$ then $I_{B} \hat{\beta}_{t}(D)=I_{B} \hat{\beta}_{t}\left(D^{\prime}\right)$

2. $\operatorname{ess}_{\inf } \hat{\beta}_{t}(D)=z_{d}, \operatorname{ess} \sup _{D} \hat{\beta}_{t}(D)=z_{u}$

3. non decreasing monotonicity: if $D_{r} \geq D_{r}^{\prime}$ for all $r \geq t$, then $\hat{\beta}_{t}(D) \geq \hat{\beta}_{t}\left(D^{\prime}\right)$;

4. strict monotonicity over constant positive shifts, i.e.

$$
c>0, r \geq t \Rightarrow \hat{\beta}_{t}\left(D+c I_{\left\{r^{*}\right\}}\right)>\hat{\beta}_{t}(D) \text { on }\left\{\hat{\beta}_{t}\left(D+c I_{\left\{r^{*}\right\}}\right)<z_{u}\right\} \cap\left\{\hat{\beta}_{t}(D+c)>z_{d}\right\}
$$

5. quasi concavity: for any $c \in[0,1]$ and for all $D, D^{\prime} \in \mathcal{D}$

$$
\hat{\beta}_{t}\left(c D+(1-c) D^{\prime}\right) \geq \min \left(\hat{\beta}_{t}(D), \hat{\beta}_{t}\left(D^{\prime}\right)\right)
$$

6. translation invariance: for all $D \in \mathcal{D}$ and $\xi \in L_{t}^{b b}$, measurable bounded from below, for all $r \geq t$

$$
\hat{\beta}_{t}\left(D+\xi I_{\{t\}}\right)=\hat{\beta}_{t}\left(D+\xi I_{\{r\}}\right)
$$

7. scale invariance, if $\beta_{t}$ is a CAI.

A map $\alpha_{t}$ on $\mathcal{D}$ with the above properties will be called a CPM for processes (a CAI for processes if scale invariant). In turn any $\alpha_{t}$ induces a CPM $\beta_{t}^{*}$ on $L_{T}^{b b}$ by the formula

$$
\beta_{t}^{*}(X)=\alpha_{t}\left(X I_{\{T\}}\right)
$$

i.e. by restricting $\alpha_{t}$ to dividend processes with a single cashflow at the terminal date $T$. The relation

$$
\left(\hat{\beta}_{t}\right)^{*}=\beta_{t},
$$

is clear, while in general $\widehat{\alpha_{t}^{*}} \neq \alpha_{t}$ unless $\alpha_{t}$ verifies (15).

A DPM for dividend processes is then a collection $\alpha=\left(\alpha_{t}\right)_{t \in \mathcal{T}}$ of CPMs with $\alpha_{t}(+\infty)=$ $z_{u}, \alpha_{t}(-\infty):=\lim _{n} \alpha_{t}(-n)=z_{d}$ for all $t$. The time consistency property for these DPMs is formulated as the analog of time consistency for DPM on variables:

Definition 4.16. The DPM for processes $\alpha$ is time consistent if

$$
\alpha_{t}(D)>z \Rightarrow \alpha_{s}(D)>z \text { for all } D \in \mathcal{D}, z_{d}<z<z_{u}
$$

We conclude by noting that a DPM $\beta$ is time consistent if and only if the induced DPM for processes $\hat{\beta}$ is time consistent. The simple proof is left to the reader.

Remark 4.17. In the same way, one can show there is a correspondence between conditional/dynamic risk measures on dividend processes and conditional/dynamic risk measures on random variables. 


\section{References}

[1] B. Acciao and I. Penner. Dynamic convex risk measures. In G. Di Nunno and B. Öksendal (Eds.), Advanced Mathematical Methods for Finance, Springer 2011, pp. 1-34.

[2] C. D. Aliprantis and K. C. Border. Infinite dimensional analysis, 3rd Edition, Springer, 2005.

[3] A.E. Bernardo and O. Ledoit. Gain, Loss and Asset Pricing. Journal of Political Economy, 108/1, 144-172, 2000.

[4] S. Biagini and M. Pinar. The Best Gain-Loss Ratio is a Poor Performance Measure. Preprint 2012, available on Arxiv (arXiv:1209.6439)

[5] S. Biagini and M. Frittelli. On the extension of the Namioka-Klee theorem and on the Fatou property for Risk Measures. in: Optimality and risk: modern trends in mathematical finance. The Kabanov Festschrift. Editors: F. Delbaen, M. Rasonyi, Ch. Stricker. Springer 2010, pp 1-28.

[6] T. R. Bielecki, I. Cialenco and Zhao Zhang. Dynamic Coherent Acceptability Indices and their Applications to Finance. Forthcoming in Mathematical Finance.

[7] T. R. Bielecki, I. Cialenco, I. Iyigunler and R. Rodriguez. Dynamic Conic Finance: Pricing and Hedging via Dynamic Coherent Acceptability Indices with Transaction Costs, preprint.

[8] J. Bion-Nadal. Conditional risk measures and robust representation of convex conditional risk measures. Ecole Polytechnique CMAP preprint 557, 2004.

[9] J. Bion-Nadal. Dynamic risk measures: Time consistency and risk measures from BMO martingales. Finance and Stochastics, 12 (2008), 219-244.

[10] J. Bion-Nadal. Time consistent dynamic risk processes. Stochastic processes and their Applications, 119 (2009), 633-654.

[11] J. Bion-Nadal Time consistent convex Feller processes and non linear second order partial differential equations preprint arXiv:1207.1742

[12] A. Cerný. Generalized Sharpe Ratios and Asset Pricing in Incomplete Markets. Eur. Finance Rev. 7 (2003), 191-203.

Electronic Journal of Probability, 11 (2006), 57-106

[13] S. Drapeau and M. Kupper. Risk preferences and their robust representation, preprint (2010).

[14] A. S. Cherny and M. Kupper. Divergence utilities, preprint (2007).

[15] A. S. Cherny and D.B. Madan. New measures for performance evaluation. Review of Financial Studies, 22 (2009), No. 7, 2571-2606. 
[16] K. Detlefsen and G. Scandolo, Conditional and dynamic convex risk measures, Finance and stochastics $9,2005,539-561$.

[17] H. Foellmer and A. Schied. Stochastic Finance, 2nd Edition. De Gruyter, 2004.

[18] M. Frittelli and M. Maggis. Conditional Certainty Equivalent. IJTAF, 14/1, 41-59, 2011.

[19] H. Gerber. An Introdution to Mathematical Risk Theory. S.S. Huebner Foundationor Insurance Education, Wharton School, University of Pennsylvania, Philadelphia, 1979.

[20] M. Kupper and W. Schachermayer. Representation Results for Law Invariant Time Consistent Functions. Mathematics and Financial Economics, 2/3, 189-210, 2009.

[21] J. Leitner. Optimal portfolios with lower partial moment constraints and lpm-risk-optimal martingale measures. Math. Fin. Volume 18/2, 317-331, 2008.

[22] J. Neveu, Discrete Parameter Martingales, North Holland, 1974.

[23] S. Peng, Backward SDE and related g-expectations. In: Backward stochastic differential equations (N. El Karoui and L. Mazliak eds.), Pitman Research Notes in Mathematics Series Vol. 364, Longman, Harlow, 141-159, 1997.

[24] E. Rosazza Gianin and C. Sgarra. Acceptability Indexes Via 'G-Expectations': An Application to Liquidity Risk, preprint. 